\title{
ON THE COMPLETE MONOTONICITY OF QUOTIENT OF GAMMA FUNCTIONS
}

\author{
Yi-Chao Chen, Toufik Mansour and Qian Zou
}

Abstract. In this paper, we concern with a conjecture involving the ratio of two gamma functions posed by Qi, Guo and Chen [Math. Inequal. Appl. 9(3)(2006), 427-436]. We also generalize a result of Qi [Theorem 2, J. Comput. Appl. Math. 214 (2008), 610-616].

Mathematics subject classification (2010): 33B15, 26A48, 26A51.

Keywords and phrases: gamma function, psi function, completely monotonic function, logarithmically completely monotonic function.

\section{REFERENCES}

[1] M. Abramowitz and LA. Stegun, Handbook of Mathematical Functions, New York, Dover, 1965.

[2] H. Alzer AND C. Berg, Some classes of completely monotonic functions II, Ramanujan J. 11 (2006), 225-248.

[3] N. BATIR, On some properties of digamma and polygamma functions, J. Math. Anal. Appl. 328 (2007), 452-465.

[4] N. Elezović, C. Giordano And J. PeČarić, The best bounds in Gautschi's inequality, Math. Inequal. Appl. 3 (2000), 239-252.

[5] B.-N. Guo AND F. QI, An Alternative Proof of Elezović-Giordano-Pečarić's Theorem, Math. Inequal. Appl., to appear.

[6] V. Krasniqi, T. Mansour And A. Sh. Shabani, Some monotonicity properties and inequalities for $\Gamma$ and $\zeta$ - functions, Math. Communications 15, 2 (2010), 365-376.

[7] V. KRASNiQI AND A. Sh. Shabani, Convexity properties and inequalities for a generalized gamma function, Appl. Math. E-Notes 10 (2010), 27-35.

[8] W. Maguns, F. Oberhettinger And R.P. Soni, Formulas and Theorems for the special Functions of Mathematical Physics, Berlin:Springer-Verlag, 1966.

[9] M. MERKLE, Inequalities for the gamma function via convexity, In: P. Cerone, S.S. Dragomir (Eds.), "Advances in Inequalities for Special Functions", Nova Science Publishers, New York, 2008, 81-100.

[10] F. QI, Three classes of logarithmically completely monotonic functions involving gamma and psi functions, Integral Transforms Spec. Funct. 18 (2007), 503-509.

[11] F. QI, A new lower bound in the second Kershaw's double inequality, J. Comput. Appl. Math. 214 (2008), 610-616.

[12] F. QI, Bounds for the ratio of two gamma functions, J. Inequal. Appl. (2010), Article ID 493058.

[13] F. QI AND CH.-P. CHEN, Monotonicity and convexity results for functions involving the gamma function, Int. J. Appl. Math. Sci. (2004), 27-36.

[14] F. QI AND SH.-X. Chen, Complete monotonicity of the logarithmic mean, Math. Inequal. Appl. 10 (2007), 799-804.

[15] F. QI AND B.-N. GuO, Wendel's and Gautschi's inequalities: Refinements, extensions, and a class of logarithmically completely monotonic functions, Appl. Math. Comput. 205 (2008), 281-290.

[16] F. QI, B.-N. GuO And Ch.-P. Chen, The best bounds in Gautschi-Kershaw inequalities, Math. Inequal. Appl. 9 (2006), 427-436.

[17] F. QI, B.-N. Guo AND CH.-P. ChEn, Some completely monotonic functions involving the gamma and polygamma functions, J. Austral. Math. Soc. 79 (2006), 31-36. 
[18] F. QI, S. GUO AND SH.-X. CHEn, A new upper bound in the second Kershaw's double inequality and its generalizations, J. Comput. Appl. Math. 220 (2008), 111-118. 